\title{
Abnormal sonographic appearances of the yolk sac: which can be associated with adverse perinatal outcome?
}

\author{
Sinan Tan ${ }^{1}$, Nuray Gülden Tangal ${ }^{1}$, Mine Kanat-Pektas², Ayşenur Şirin Özcan'1, \\ Hüseyin Levent Keskin ${ }^{3}$, Gökçe Akgündüz ${ }^{1}$, Mehmet Akif Teber ${ }^{4}$, Halil Arslan ${ }^{1}$
}

${ }^{1}$ Ankara Atatürk Education and Research Hospital, Department of Radiology, ${ }^{2}$ Afyon Kocatepe University Medical Faculty Hospital, Department of Obstetrics and Gynecology, ${ }^{3}$ Ankara Atatürk Education and Research Hospital, Department of Obstetrics and Gynecology, ${ }^{4}$ Etlik Ihtisas Research and Education Hospital, Department of Radiology, Turkey

\begin{abstract}
Aims: The present study aimed to determine whether yolk sacs with abnormal sonographic appearance are associated with adverse perinatal outcomes in both early and late gestation. Matherial and methods: A total of 305 viable singleton pregnancies with gestational age of 6 to 9 weeks were prospectively evaluated with respect to perinatal outcomes and sonographic characteristics of the yolk sacs. Results: An abnormal yolk sac was found in 66 pregnancies. In pregnancies with enlarged yolk sacs a miscarriage occurred in $37.5 \%$ of cases (3/8). The pregnancies with a yolk sac diameter $\geq 5$ mm had a significantly higher risk of miscarriage $(\mathrm{p}=0.005)$. The risk of miscarriage was statistically similar between the pregnancies with regular and those with irregular yolk sacs $(\mathrm{p}=0.73)$. Miscarriage occurred in $3.8 \%$ of pregnancies with irregular yolk sacs $(2 / 52)$ and none of pregnancies with echogenic yolk sacs $(0 / 6)$. Adverse perinatal outcomes were not associated with either irregular or echogenic yolk sacs. Conclusions: An enlarged yolk sac visualized before the 7th week of gestation is strongly associated with a significantly increased risk for spontaneous miscarriage. The presence of an echogenic or irregular yolk sac appears to be unrelated to adverse perinatal outcome.
\end{abstract}

Keywords: yolk sac, pregnancy, ultrasound, first trimester, miscarriage.

\section{Introduction}

The yolk sac acts as the primary route of exchange between the human embryo and the mother before the placental circulation is established. The yolk sac provides nutritional, metabolic, endocrine, immunologic, and hematopoietic functions during organogenesis in embryonic life, and is considered to reach its highest level of functional activity between the $4^{\text {th }}$ and $7^{\text {th }}$ week of embryonic development [1].

Received 29.10.2013 Accepted 11.12.2013

Med Ultrason

2014, Vol. 16, No 1, 15-20

Corresponding author: Sinan Tan, MD

Ankara Atatürk Education and Research Hospital, Radiology Department

Eskişehir Yolu 8.km No: 3 Bilkent, 06800, Ankara, Turkey

Phone: +90(312) 2912525 Ext: 4620

Fax: +90(312) 2912705

E-mail: drsinantan@gmail.com
The yolk sac is a critical landmark that identifies a true gestational sac [2]. In fact, it is the first conceptional structure that is usually visualized by transvaginal sonography when a gestational sac measures over $8 \mathrm{~mm}$. Sonography shows the yolk sac as a round structure that is made up of an anechoic center bordered by a regular well-defined echogenic rim. The diameter of a yolk sac is usually 3-4 mm and increases in size up to the $10^{\text {th }}$ or $11^{\text {th }}$ week of gestation [3].

It has been hypothesized that abnormal sonographic findings related to the size, shape and internal structure of a yolk sac can be used to predict gestational outcome [3-7]. It has been well established that an abnormally large yolk sac correlates with early pregnancy failure $[4,6,7]$. On the other hand, studies focusing on the shape or internal structure of the yolk sac have yielded conflicting results. Some studies suggest that irregular yolk sac shape and echogenic yolk sac can be associated with fetal death or abnormalities [5,8-10]. In contrast, some authors claimed that a pregnancy could have a completely 
normal course even in the presence of irregular shape or echogenic yolk sac $[11,12]$. Therefore, its effects on gestational outcome required further investigation.

The present study aimed to determine whether yolk sacs with an abnormal sonographic appearance in pregnancies at 6 to 9 weeks of gestation are associated with adverse perinatal outcomes in both early and late gestation.

\section{Matherial and methods}

\section{Study design and patients}

This prospective observational study was approved by the institutional Review Board from our institution, and informed written consent was obtained from each participant. The present study reviewed 354 viable singleton pregnancies with a gestational age of 6 to 9 weeks that were consecutively examined by the Radiology Department of the study center as part of routine follow-up between March 2011 and January 2012. In order to avoid any confounding or biased results as these conditions may interfere with adverse pregnancy outcome, 19 pregnancies with large subchorionic hemorrhage, embryonic bradycardia, chorionic bump or uterine fibroids were excluded.

\section{Equipment and Scanning}

All pregnancies were assessed by LOGIQ 9 sonographic equipment with a 5- to 8-MHz multifrequency transvaginal transducer (GE Healthcare, Milwaukee, WI, USA). Sonographic examinations were performed by two of three radiologists (S.T., A.O, and G.T) with 3-7 years experience in obstetric sonography so that conclusions could be drawn by consensus. All of the sonographic examinations were done while the urinary bladder was empty and acquired images were recorded digitally on the local sonography device.

Gestational age was calculated according to the embryonic crown-rump length. Calipers were used to measure the internal diameter of the yolk sac on a magnified scan and both the shape and appearance of this structure was evaluated (fig 1). During the sonography examinations, we carefully evaluated potential artifacts such as partial volume and harmonics, because these artifacts may lead to misinterpretation of yolk sac structure. Therefore, adjustments of the ultrasound image, such as

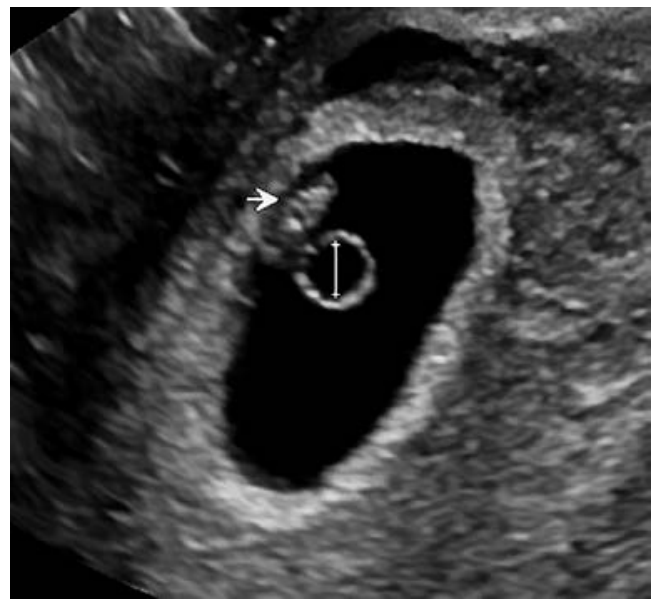

Fig 1. Normal yolk sac. Transvaginal sonography shows a living embryo (arrow) at 6 weeks 2 days and a yolk sac with an anechoic center bordered by smooth and non-deformed margins. The calipers are placed at the inner edges of the yolk sac wall.
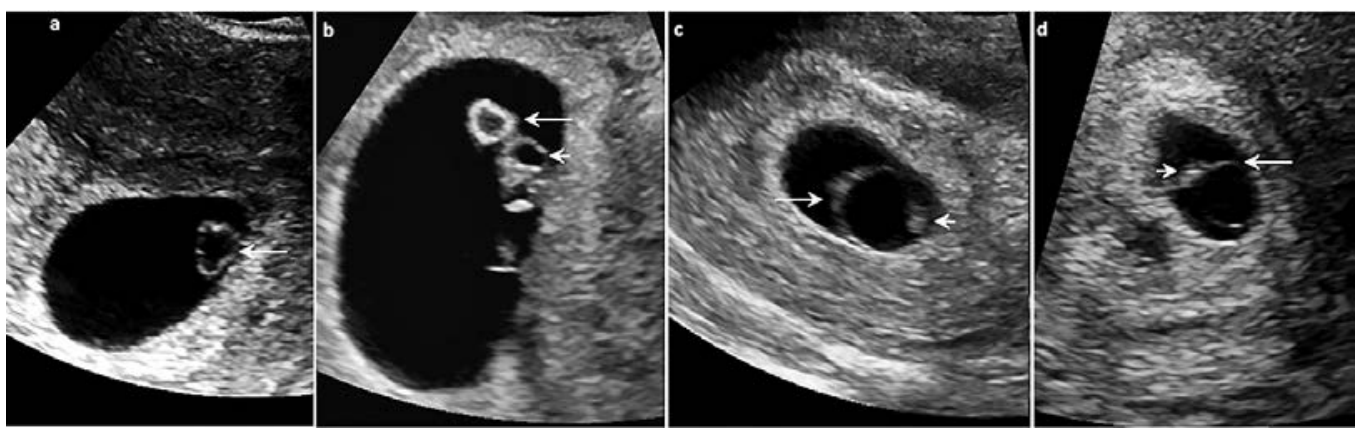

Fig 2. a-d. Sonographic appearance of the abnormal yolk sac, transvaginal sonography: (a) of a live embryo at 6 weeks 1 day shows a yolk sac with an irregular shape (arrow); (b) at 7 weeks 3 days shows a live embryo and an echogenic yolk sac (long arrow). Small arrow denotes the cystic rhombencephalon; (c) at 6 weeks 0 days shows a live embryo (small arrow) and a large yolk sac (long arrow) with a mean diameter of $7.2 \mathrm{~mm}$. This pregnancy continued uneventfully until delivery at 40 weeks gestation with a good neonatal outcome; (d) at 6 weeks 0 days shows a live embryo (small arrow) and a large yolk sac (long arrow) with a mean diameter of $5 \mathrm{~mm}$. In this case, despite normal sonographic cardiac activity, a miscarriage occurred on a follow-up examination two weeks later. 
insonation angle, focal zone and gray-scale gain setting for accurate interpretation of yolk sac structure was adjusted, and tissue harmonic imaging was utilized.

The yolk sac with wrinkled margins, indented walls, or both was defined as having an irregular shape. If the internal structure of a yolk sac had echogenicity rather than being totally anechoic, it was described as an echogenic yolk sac. A large yolk sac was defined as a yolk sac with a diameter $\geq 5 \mathrm{~mm}$ (fig 2).

\section{Clinical Follow Up}

A total of 66 pregnancies with an abnormal appearing yolk sac were re-evaluated by sonography after two weeks. All of the recruited pregnancies were scheduled for nuchal translucency measurement between the $11^{\text {th }}$ and $14^{\text {th }}$ week of gestation and fetal anomaly scan was undertaken between the $18^{\text {th }}$ and $22^{\text {nd }}$ week of gestation. All pregnancies were followed up until the time of delivery. Data related with perinatal outcomes were obtained from medical records of the study center. As for 28 pregnancies that ended up with delivery outside the study center, data related to perinatal outcomes were acquired by telephone interviews. Since 30 pregnancies were excluded because of loss to follow up, the remaining 305 pregnancies were enrolled for final analysis.

An adverse perinatal outcome was defined as either perinatal mortality (spontaneous pregnancy loss during the first trimester of pregnancy or intrauterine fetal demise) or perinatal morbidity (such as first and second trimester abnormal sonographic findings, isolated structural defects and gestational problems). Gestational problems associated with perinatal morbidity account for polyhydramnios, oligohydramnios, preeclampsia, gestational diabetes, preterm delivery (24-36 weeks gestation), hyperthyroidism and cholestasis of pregnancy.

\section{Statistical analysis}

Collected data were analyzed by the Statistical Package for Social Sciences on computerized media (SPSS version 16.0, SPSS Inc, Chicago, IL, USA). Continuous variables were expressed as either mean \pm standard deviation (SD) or percentage, where appropriate. Spearman correlation test, chi square test and Fisher's exact test were utilized to detect the correlations between the variables whereas the odds ratios and corresponding $95 \%$ confidence intervals were used to estimate miscarriage risks. Two tailed $\mathrm{p}$ values $<0.05$ were accepted to be statistically significant.

\section{Results}

Table I lists the clinical characteristics of the 305 pregnancies reviewed in the present study. The mean yolk sac diameter was $3.2 \pm 0.7 \mathrm{~mm}$ (range: $2.0-7.2 \mathrm{~mm}$ ). There was a moderately positive correlation between yolk sac size and gestational age and yolk sac diameter increasing progressively with advancing gestational weeks $(r=$ $0.51, \mathrm{p}<0.001)$.

About $78.4 \%$ of the reviewed pregnancies (239 of $305)$ had normally appearing yolk sacs, whereas $21.6 \%$ of them (66 of 305) had abnormally appearing yolk sacs (table II). An irregular yolk sac was observed in 52 / 66 (78.8\%), an enlarged yolk sac in $8 / 66(12.1 \%)$, and an echogenic yolk sac in 6 / 66 (9.1\%). Miscarriage occurred in $37.5 \%$ of pregnancies with enlarged yolk sacs $(3 / 8)$, $3.8 \%$ of pregnancies with irregular yolk sacs $(2 / 52)$ and none of the pregnancies with echogenic yolk sacs. Missed abortion was already present in two pregnancies with enlarged yolk sacs and one pregnancy with irregular yolk sac and none of the pregnancies with echogenic yolk sacs.

Table I. Clinical characteristics of the reviewed pregnancies.

\begin{tabular}{lccc}
\hline Characteristic & Mean \pm SD & Median & Min-Max \\
\hline Maternal age (years) & $26.5 \pm 4.9$ & 26 & $18-42$ \\
Gravidity & $1.9 \pm 1.1$ & 2 & $1-6$ \\
Parity & $1.6 \pm 0.9$ & 1 & $0-4$ \\
Gestational age at delivery (weeks) & $38.9 \pm 1.9$ & 39 & $30-42$ \\
Neonatal birth weight (grams) & $3247.3 \pm 511.1$ & 3200 & $1500-4700$ \\
\hline
\end{tabular}

Table II. Yolk sac shape and size according to gestational week.

\begin{tabular}{|c|c|c|c|c|c|}
\hline \multirow[t]{2}{*}{ Gestational age } & \multicolumn{4}{|c|}{ Yolk sac shape } & \multirow{2}{*}{$\begin{array}{c}\text { Yolk sac diameter }(\mathbf{m m}) \\
\text { Mean } \pm \text { SD (Min-Max) }\end{array}$} \\
\hline & Normal & Irregular & Echogenic & Enlarged & \\
\hline 6 th week $(n=129)$ & $90(69.8 \%)$ & $30(23.2 \%)$ & $4(3.1 \%)$ & $5(\% 3.9)$ & $2.9 \pm 0.73(2.0-7.2)$ \\
\hline 7 th week $(n=82)$ & $63(76.9 \%)$ & $18(21.9 \%)$ & $1(1.2 \%)$ & $0(0.0 \%)$ & $3.1 \pm 0.53(2.0-4.6)$ \\
\hline 8 th week $(n=55)$ & $50(90.9 \%)$ & $4(7.3 \%)$ & $1(1.8 \%)$ & $0(0.0 \%)$ & $3.4 \pm 0.73(2.7-4.8)$ \\
\hline 9 th week $(n=39)$ & $36(92.3 \%)$ & $0(0.0 \%)$ & $0(0.0 \%)$ & $3(\% 7.7)$ & $4.1 \pm 0.73(2.7-5.6)$ \\
\hline
\end{tabular}


Table III. Adverse perinatal outcome according to yolk sac characteristics.

\begin{tabular}{|c|c|c|c|c|}
\hline Perinatal Outcome & $\begin{array}{l}\text { Normal } \\
\text { yolk sac }\end{array}$ & $\begin{array}{c}\text { Irregular } \\
\text { yolk sac }\end{array}$ & $\begin{array}{c}\text { Enlarged } \\
\text { yolk sac }\end{array}$ & $\begin{array}{c}\text { Echogenic } \\
\text { yolk sac }\end{array}$ \\
\hline \multicolumn{5}{|l|}{ Perinatal morbidity } \\
\hline \multicolumn{5}{|l|}{ Abnormal sonographic findings } \\
\hline Umbilical cord cyst $\dagger$ & 1 & - & - & - \\
\hline Echogenic intracardiac focust & 3 & 1 & - & 1 \\
\hline Mild ventriculomegaly $\$$ & 1 & - & - & - \\
\hline Mild pyelectasist & 3 & - & 1 & - \\
\hline Choroid plexus cysts & 2 & - & - & - \\
\hline Increased nuchal fold thickness: & 1 & 1 & - & - \\
\hline Omphalocele $\dagger$ & 1 & - & - & - \\
\hline Cleft lipt & - & 1 & - & - \\
\hline \multicolumn{5}{|l|}{ Isolated Structural Defects } \\
\hline Varus deformity & - & - & 1 & - \\
\hline Single umbilical artery & 2 & - & - & - \\
\hline Fallot tetralogy & 1 & - & - & - \\
\hline \multicolumn{5}{|l|}{ Gestational Problems } \\
\hline Polyhydramnios & 9 & 1 & - & - \\
\hline Oligohydramnios & 6 & 3 & - & - \\
\hline Preeclampsia & 5 & - & - & - \\
\hline Gestational diabetes & 9 & 1 & - & - \\
\hline Preterm delivery & 15 & 4 & - & - \\
\hline Hypertyroidism & - & 1 & - & - \\
\hline Cholestasis of pregnancy & 2 & - & - & - \\
\hline \multicolumn{5}{|l|}{ Perinatal Mortality } \\
\hline Missed abortion $\dagger$ & 2 & 1 & 2 & - \\
\hline Spontaneous miscarriage $\dagger$ & 8 & 1 & 1 & - \\
\hline Intrauterine fetal demise & 5 & - & - & - \\
\hline
\end{tabular}

$\dagger$ These abnormalities are detected by ultrasonography screening during the first trimester.

† These abnormalities are detected by ultrasonography at mid-second trimester.

Three pregnancies with an enlarged yolk sac (diameters of $6.1 \mathrm{~mm}, 5.9$ and $5 \mathrm{~mm}$ ) resulted in first-trimester miscarriage. All of these cases were visualized before the $7^{\text {th }}$ week of gestation. The remaining five pregnancies (with diameters of 7.2, 5.6, 5.2, 5.1, and $5 \mathrm{~mm}$ ) continued seamlessly. When compared with pregnancies that had a yolk sac diameter $<5 \mathrm{~mm}$, the pregnancies with a yolk sac diameter $\geq 5 \mathrm{~mm}$ had a significantly higher risk of miscarriage $(\mathrm{p}=0.005$; Odds ratio: $14.25 ; 95 \%$ confidence interval: 3-66.7). If such a comparison had been made for pregnancies with a gestational age of 6 weeks, the miscarriage risk would have been even more significant $(\mathrm{p}=0.002$; Odds ratio: 29.5 ; $95 \%$ confidence interval: 4.1-211.2).

Two pregnancies with an irregular yolk sac ended up with a miscarriage before the $10^{\text {th }}$ week of gestation, while the remaining 50 cases continued with normal appearing yolk sacs. The risk of miscarriage was statistically similar between the pregnancies with regular yolk sacs and those with irregular yolk sacs $(\mathrm{p}=0.73)$. As gestational age advanced, the frequency of an irregular yolk sac decreased significantly $(p=0.001)$.
An echogenic yolk sac was detected in six pregnancies. All of these pregnancies showing a normal appearing yolk sac underwent a second sonographic examination after 2 weeks and continued uneventfully until the $40^{\text {th }}$ gestational week. Table III shows that adverse perinatal outcomes were not associated with either irregular or echogenic yolk sacs.

\section{Discussions}

Sequential appearance of a yolk sac, embryonic heartbeat, and an amniotic cavity is essential for normal pregnancy [5]. The yolk sac is seen in all pregnancies from 5 weeks' gestation onwards, when the gestational sac exceeds $11 \mathrm{~mm}$ in diameter [13]. Usual sonographic appearance of a yolk sac has been defined as a 3 to 5 $\mathrm{mm}$ wide anechoic round structure that is encircled by a regular echogenic rim. It has been accepted that the diameter of a yolk sac normally increases by $0.1 \mathrm{~mm}$ every 24 hours and undergoes rapid degeneration at the end of the $10^{\text {th }}$ or $11^{\text {th }}$ week of pregnancy [4]. 
Recently, Bagratee et al. demonstrated that the reference intervals for first-trimester yolk sac volume increased in a linear fashion up to 10 weeks, then maintained a plateau until 11 weeks and decreased afterwards [14].

The decreased vascularity of the yolk sac at the time of its maximum volume is proposed as the cause of its degeneration and disappearance. That is, the disappearance of arterial signals in the yolk sac circulation and a simultaneous increase in the umbilicoplacental blood flow indicates that the transition from the yolk sac to the placenta occurs as an essential source of blood supply to the embryo between $8^{\text {th }}$ and $10^{\text {th }}$ weeks of gestation [15].

Despite the fact that there is no clearly identified consensus, an enlarged yolk sac can be depicted as a yolk sac with a diameter of 5 or $6 \mathrm{~mm}$. Generally, it has been suggested that an abnormally large yolk sac indicates poor obstetric outcome $[4,6,7]$. A recent study has shown that a yolk sac diameter of greater than $5 \mathrm{~mm}$ is associated with an increased risk of spontaneous abortion [6]. However, a few authors have mentioned the existence of a very large yolk sac (with a diameter of $8.1 \mathrm{~mm}$ ) in a normal live pregnancy [5]. In our study, an enlarged yolk sac was noted in eight pregnancies $(2.6 \%)$. Nearly $40 \%$ of these pregnancies resulted in first-trimester miscarriage. When compared with pregnancies that had yolk sac diameter $<5 \mathrm{~mm}$, the pregnancies with yolk sac diameter $\geq 5 \mathrm{~mm}$ had a significantly higher risk of miscarriage (p $=0.005$ ). If such a comparison was made for pregnancies with a gestational age of 6 weeks, the miscarriage risk would be even more significantly higher $(\mathrm{p}=0.002)$. These findings indicate that the existence of an enlarged yolk sac (with a diameter of $\geq 5 \mathrm{~mm}$ ) is of evident clinical significance when it is specified before the $7^{\text {th }}$ week of gestation.

The literature contains conclusions about the clinical importance of an abnormal yolk sac shape that are controversial and thus, there is still an ongoing debate. Some clinical studies have declared that the persistence of an irregular yolk sac shape may be used to indicate an adverse gestational outcome $[3,4]$. However, a newly published study suggests that an irregular yolk sac shape is not associated with an increased risk of spontaneous abortion [12]. In our study, an irregular yolk sac was observed in 52 pregnancies (17.1\%) and approximately $4 \%$ of them ended up with a miscarriage before the $10^{\text {th }}$ week of gestation. The detection of an irregular yolk sac did not significantly change the miscarriage risk and was found to be unrelated to adverse perinatal outcome.

An echogenic yolk sac is visualized as a bright yolk sac that may present with various types of materials other than calcium. An important issue to be emphasized here is that an echogenic yolk sac should be distinguished from a calcified one. The reason is that an echogenic yolk sac can be visualized in normal pregnancies while a calcified yolk sac is almost always associated with embryonic demise [11]. Tissue harmonic imaging can be utilized to detect and confirm the existence of echogenic yolk sacs [2].

To the best of our knowledge, only two studies have reported that an echogenic yolk sac can be associated with fetal death or abnormalities $[5,8]$. On the other hand, Levi and Lyons declare that an echogenic yolk sac is not always associated with anomalies or impending demise and may revert to a more normal appearance within a week [11]. Only one study has followed the pregnancies with echogenic yolk sacs alone and in conjunction with nuchal translucency in 3620 first trimester pregnancies. Accordingly, 39 cases (1\%) of echogenic yolk sac were detected among pregnancies with a gestational age of 9 to 11 weeks. Nineteen cases $(0.5 \%)$ had both a nuchal translucency of $>3 \mathrm{~mm}$ and an echogenic yolk sac, and all were chromosomally abnormal. Twenty cases $(0.5 \%)$ had an echogenic yolk sac as the only unusual finding and all were chromosomally normal [8]. In our study, an echogenic yolk sac was detected in six pregnancies $(1.9 \%)$, which turned out to have normal yolk sacs before the $10^{\text {th }}$ gestational week. Moreover, the presence of an echogenic yolk sac was found to be unrelated to adverse perinatal outcome. However, large-scale prospective studies are anticipated to clarify the prognostic importance of echogenic yolk sacs.

The present study prospectively evaluates a relatively large number or pregnancies and offers a longitudinal scan for pregnancies with both normal and abnormal yolk sacs. Although these factors may provide some advantages, there are two factors that limit the power of the findings of the present study. First, this study ignores several factors that may interfere with the course of pregnancy (e.g. smoking, obesity, polycystic ovary syndrome). Second, this study reviews a relatively small number of pregnancies with echogenic or enlarged yolk sacs.

\section{Conclusions}

An enlarged yolk sac visualized before the $7^{\text {th }}$ week of gestation is strongly associated with a significantly increased risk for spontaneous miscarriage. Therefore, any pregnancy that is sonographically identified with an enlarged yolk sac should be monitored closely. The presence of an echogenic or irregular yolk sac appears to be unrelated to adverse perinatal outcome. In addition, as gestational age advances, these abnormalities in the sonographic appearance of a yolk sac are encountered significantly less often. We think that both irregular and 
echogenic yolk sacs may be related to the physiological findings in the early first trimester.

\section{Conflict of interest: none}

\section{References}

1. Pereda J, Niimi G. Embryonic erythropoiesis in human yolk sac: two different compartments for two different processes. Microsc Res Tech 2008; 71: 856-862.

2. Kurtz AB, Needleman L, Pennell RG, Baltarowich O, Vilaro $\mathrm{M}$, Goldberg BB. Can detection of the yolk sac in the first trimester be used to predict the outcome of pregnancy? A prospective sonographic study. AJR Am J Roentgenol 1992; 158: 843-847.

3. Küçük T, Duru NK, Yenen MC, Dede M, Ergün A, Başer I. Yolk sac size and shape as predictors of poor pregnancy outcome. J Perinat Med 1999; 27: 316-320.

4. Lindsay DJ, Lovett IS, Lyons EA, et al. Yolk sac diameter and shape at endovaginal US: predictors of pregnancy outcome in the first trimester. Radiology 1992; 183: 115-118.

5. Cho FN, Chen SN, Tai MH, Yang TL. The quality and size of yolk sac in early pregnancy loss. Aust N Z J Obstet Gynaecol 2006; 46: 413-418.

6. Berdahl DM, Blaine J, Van Voorhis B, Dokras A. Detection of enlarged yolk sac on early ultrasound is associated with adverse pregnancy outcomes. Fertil Steril 2010; 94: 15351537.
7. Bae S, Karnitis J. Triple ultrasound markers including fetal cardiac activity are related to miscarriage risk. Fertil Steril 2011; 96: 1145-1148.

8. Szabo J, Gellen J, Szemere G, Farago M. Significance of hyper-echogenic yolk sac in first-trimester screening for chromosome aneuploidy. Orv Hetil 1996; 137: 2313-2315.

9. Parasuraman R, Liversedge HM, Gilg J, Taylor MJ. Echogenic yolk sac: a marker for aneuploidy? J Obstet Gynaecol 2009; 29: 405-406.

10. Schmidt P, Hörmansdörfer C, Bosselmann S, Elsässer M, Scharf A. Is the yolk sac a new marker for chromosomal abnormalities in early pregnancy? Arch Gynecol Obstet 2011; 283: 23-26.

11. Lyons EA, Levi CS. The first trimester. In: Rumack CM, Wilson SR, Charboneau JW (eds). Diagnostic Ultrasound. 3rd ed. St Louis: Mosby 2005: 1070-1100.

12. Tan S, Ípek A, Pektas MK, Arifoğlu M, Teber MA, Karaoğlanoğlu M. Irregular yolk sac shape: is it really associated with an increased risk of spontaneous abortion? J Ultrasound Med 2011; 30: 31-36.

13. Bree RL, Marn CS. Transvaginal sonography in the first trimester: embryology, anatomy and hCG correlation. Semin Ultrasound CT MR 1990; 11: 12-21.

14. Bagratee JS, Regan L, Khullar V, Connolly C, Moodley J. Reference intervals of gestational sac, yolk sac and embryo volumes using three-dimensional ultrasound. Ultrasound Obstet Gynecol 2009; 34: 503-509.

15. Kupesic S, Kurjak A, Ivancic-Kosuta M. Volume and vascularity of the yolk sac studied by three-dimensional ultrasound and color Doppler. J Perinat Med 1999; 27: 91-96. 